\title{
Effects of metformin on mitochondrial function of leukocytes from polycystic ovary syndrome patients with insulin resistance
}

\author{
Victor M Victor ${ }^{1,2,3}$, Susana Rovira-Llopis ${ }^{1,2}$, Celia Bañuls ${ }^{1,2}$, Noelia Diaz-Morales', \\ Raquel Castelló ${ }^{1}$, Rosa Falcón', Marcelino Gómez ${ }^{1}$, Milagros Rocha ${ }^{1,2,3}$ and \\ Antonio Hernández-Mijares ${ }^{1,2,4}$
}

${ }^{1}$ Service of Endocrinology, University Hospital Doctor Peset, Foundation for the Promotion of Health and Biomedical Research in the Valencian Region (FISABIO), Avenida Gaspar Aguilar 90, 46017 Valencia, Spain, ${ }^{2}$ Institute of Health Research INCLIVA, University of Valencia, Valencia, Spain, ${ }^{3} \mathrm{CIBERehd}$ - Department of Pharmacology and Physiology, University of Valencia, Valencia, Spain and ${ }^{4}$ Department of Medicine, University of Valencia, Valencia, Spain

\author{
Correspondence \\ should be addressed \\ to V M Victor or M Rocha \\ or A Hernández-Mijares \\ Emails \\ Victor.Victor@uv.es or \\ Milagros.Rocha@uv.es or \\ hernandez_antmij@gva.es
}

\begin{abstract}
Objective: Oxidative stress and mitochondrial dysfunction are implicated in polycystic ovary syndrome (PCOS). The present study assesses the effect of metformin treatment on mitochondrial function in polymorphonuclear cells from PCOS subjects. Additionally, we evaluate endocrine parameters and levels of interleukin 6 (IL6) and tumour necrosis factor alpha (TNF $\alpha$ ). Design and methods: Our study population was comprised of 35 women of reproductive age diagnosed with PCOS and treated with metformin for 12 weeks, and their corresponding controls $(n=41)$, adjusted by age and BMI. We evaluated the alteration of endocrinological and anthropometrical parameters and androgen levels. Mitochondrial $\mathrm{O}_{2}$ consumption (using a Clark-type $\mathrm{O}_{2}$ electrode), membrane potential, mitochondrial mass, and levels of reactive oxygen species (ROS) and glutathione (GSH) (by means of fluorescence microscopy) were assessed in poymorphonuclear cells. $\mathrm{H}_{2} \mathrm{O}_{2}$ was evaluated with the Amplex Red ${ }^{\mathrm{R}} \mathrm{H}_{2} \mathrm{O}_{2}$ /Peroxidase Assay kit. IL6 and TNF $\alpha$ were measured using the Luminex 200 flow analyser system. Results: Metformin had beneficial effects on patients by increasing mitochondrial $\mathrm{O}_{2}$ consumption, membrane potential, mitochondrial mass and glutathione levels, and by decreasing levels of reactive oxygen species and $\mathrm{H}_{2} \mathrm{O}_{2}$. In addition, metformin reduced glucose, follicle-stimulating hormone, IL6 and TNF $\alpha$ levels and increased dehydroepiandrosterone sulfate levels. HOMA-IR and mitochondrial function biomarkers positively correlated with ROS production $(r=0.486, P=0.025)$, GSH content $(r=0.710, P=0.049)$ and $\mathrm{H}_{2} \mathrm{O}_{2}(r=0.837, P=0.010)$, and negatively correlated with membrane potential $(r=-0.829, P=0.011)$ at baseline. These differences disappeared after metformin treatment. Conclusions: Our results demonstrate the beneficial effects of metformin treatment on mitochondrial function in leukocytes of PCOS patients.

\section{Introduction}

Polycystic ovary syndrome (PCOS) is the most common endocrinopathy in reproductive aged women, affecting around $6-20 \%$ of said population $(1,2)$. It is a metabolic disorder characterised in a high percentage of cases by insulin resistance (IR) (3), which means that patients are at a high risk of developing type 2 diabetes (4) or metabolic syndrome (5).

The cardiovascular and metabolic consequences of PCOS are varied, and so it is necessary to administer patients a multiple therapy. Metformin has been widely

Published by Bioscientifica Ltd. 
used to treat PCOS due to the way it improves IR (6). It exerts multiple actions on different tissues affected by hyperinsulinemia and/or IR, such as adipose tissue, skeletal muscle, liver, endothelium and ovaries (7), and seems to improve the long-term health outcome of women with PCOS by preventing endometrial cancer, diabetes and cardiovascular disease (8).

It has been postulated that tissue exhibiting IR (e.g. muscle) is characterised by reduced mitochondrial content, protein expression and activity of different enzymes $(9,10)$, although there is conflicting evidence with respect to this $(11,12)$. In this sense, the molecular mechanisms exerted by metformin are not altogether clear. One potential mechanism is inhibition of mitochondrial complex I $(13,14,15)$, though there are also discrepancies about this $(11,16)$. Metformin is thought to stimulate AMP kinase by inhibiting mitochondrial complex I (14), though this does not always depend on alterations in the redox state of the cell (17).

Several studies in PCOS patients have demonstrated that acute hyperglycemia and IR induce an increase in reactive oxygen species (ROS) production by peripheral blood leukocytes $(18,19)$, activate leukocyte-endothelium interactions (20) and proinflammatory transcription factor nuclear kappa B (NF- $\mathrm{B})(21)$, and increase levels of proinflammatory cytokines (22). In this way, oxidative stress has been implicated in the etiology of IR in leukocytes from PCOS patients. Contrasting results have been obtained in studies investigating the effects of metformin on oxidative stress; for example, Kocer et al. (23) demonstrated that metformin reduced oxidative stress, while Pavlovic et al. (24) showed that it increased it.

In the present study we assess the effects of metformin treatment on endocrine and anthropometrical parameters and on mitochondrial function in PCOS patients by measuring mitochondrial oxygen $\left(\mathrm{O}_{2}\right)$ consumption, membrane potential, glutathione (GSH), ROS and $\mathrm{H}_{2} \mathrm{O}_{2}$ levels, mitochondrial mass, and interleukin 6 (IL6) and tumour necrosis factor alpha (TNF $\alpha$ ) in leukocytes from said patients.

\section{Subjects and methods}

\section{Subjects}

Forty-one PCOS women of reproductive age (21-33 years old) were enrolled in the study, as were 41 controls adjusted by age and BMI. PCOS subjects were diagnosed according to the Rotterdam criteria (25), and the study was conducted by the Service of Endocrinology, University
Hospital Dr Peset, Valencia, Spain. The Rotterdam criteria for the diagnosis of PCOS are as follows: oligoovulation (cycles longer than 35 days or shorter than 26 days) (26); elevated free testosterone levels $(>0.5 \mathrm{ng} / \mathrm{dl}$; the cut-off level for free testosterone was the mean \pm 2 s.D. according to normal levels in controls); hirsutism (total FerrimanGallwey score $>7$ ); and polycystic ovaries (12 or more small (2-9 $\mathrm{mm})$ follicles in each ovary, identified by transvaginal ultrasonography). Ultrasound scans were performed and scored independentlyby one of two experienced and blinded observers. None of the subjects had galactorrhea or any endocrine or systemic disease that could have affected her reproductive physiology. None of the women reported using any medication that could have interfered with the normal function of the hypothalamicpituitary-gonadal axis during the previous semester.

Exclusion criteria were organic, malignant, haematological, infectious or inflammatory disease and a history of ischaemic heart disease, stroke or thromboembolism, diabetes mellitus, hyperlipidaemia or hypertension. An anthropometric and analytical evaluation was performed for all subjects, and weight $(\mathrm{kg})$, height $(\mathrm{m})$ and waist $(\mathrm{cm})$ were measured and body mass index $(\mathrm{BMI}=$ weight $(\mathrm{kg}) /$ height $\left.(\mathrm{m})^{2}\right)$ calculated.

Blood was collected from the antecubital vein at 0800-1000 h on the second/third day of the menstrual cycle (follicular phase) after $12 \mathrm{~h}$ of fasting. Glucose levels were measured using enzymatic techniques and a Dax-72 autoanalyzer (Bayer Diagnostic, Tarrytown, New York, USA). Insulin was measured by an enzymatic luminescence technique. Samples were immediately processed for insulin in order to avoid haemolysis and were frozen until analysis. IR was calculated by homeostasis model assessment (HOMA) using baseline glucose and insulin: $\mathrm{HOMA}=($ fasting insulin $(\mu \mathrm{U} / \mathrm{ml}) \times$ fasting glucose $(\mathrm{mmol} / \mathrm{l}) / 22.5$. Luteinizing hormone $(\mathrm{LH})$, folliclestimulating hormone (FSH) and testosterone were measured by specific RIAs. DHEAS-sulfate, sex hormone binding globulin (SHBG), androstenedione and testosterone were measured by specific chemiluminescence techniques in our hospital's Clinical Analysis Service. High-sensitive C-reactive protein (hsCRP) was quantified by a latex-enhanced immunonephelometric assay (Behring Nephelometer II, Dade Behring, Inc., Newark, DE, USA) with an intra-assay coefficient of variation of $8.7 \%$ and sensitivity of $0.01 \mathrm{mg} / 1$.

Total cholesterol and triglycerides were measured by means of enzymatic assays, and HDLc concentrations were recorded with a Beckman LX-20 autoanalyzer (Beckman Coulter, La Brea, CA, USA) using a direct method. The 
intraserial variation coefficient was $<3.5 \%$ for all determinations. LDLc concentration was calculated using the Friedewald method. Apolipoprotein (Apo) AI and $\mathrm{B}$ was determined by immunonephelometry (Dade Behring BNII, Marburg, Germany) with an intra-assay variation coefficient of $<5.5 \%$.

PCOS subjects and controls followed a normocaloric diet. PCOS subjects began treatment with metformin at a dose of $500 \mathrm{mg} /$ day, which was increased to $1000 \mathrm{mg}$ after 2 weeks and to $1500 \mathrm{mg} /$ day after a further 2 weeks. This last dose was maintained for a total of 12 weeks. Subjects returned every 2 weeks for anthropomorphic and endocrine measurements and blood sampling. Compliance was determined by interviewing the patients during these fortnightly visits.

Informed written consent was obtained from all subjects prior to participation. The study was approved by the clinical research ethics committee of the University Hospital Doctor Peset and was performed in accordance with the Helsinki declaration. This trial was registered on clinicaltrials.gov under study number NCT02302326. Four subjects were excluded and two subjects dropped out of the study for personal reasons, because of an inability or unwillingness to comply with the protocol.

\section{Cells}

Human polymononuclear leukocytes (PMNs) were obtained from citrated blood samples and incubated for 45 min with dextran (3\%). The supernatant was centrifuged at $250 \boldsymbol{g}$ for $25 \mathrm{~min}$ in Fycoll-Hypaque. Lysis buffer was added to the pellet, which was then centrifuged at room temperature $(100 \mathrm{~g}, 5 \mathrm{~min})$. PMNs were evaluated in a Scepter device (Millipore, MA, USA), washed in Hanks' balanced salt solution (HBSS) and then stored in complete RPMI media.

\section{Measurement of $\mathrm{O}_{2}$ consumption}

PMNs were resuspended $\left(5 \times 10^{6}\right.$ cells $\left./ \mathrm{ml}\right)$ in HBSS medium and placed in a gas-tight chamber. A Clark-type $\mathrm{O}_{2}$ electrode (Rank Brothers, Bottisham, UK) was used to measure $\mathrm{O}_{2}$ consumption (27). Sodium cyanide $\left(10^{-3} \mathrm{~mol} / \mathrm{l}\right)$ was employed to evaluate whether or not $\mathrm{O}_{2}$ consumption was mitochondrial (95-99\%). Measurements were obtained using the data-device Duo.18 (WPI, Stevenage, UK). The rate of $\mathrm{O}_{2}$ consumption $\left(\mathrm{V}_{\mathrm{O} 2 \mathrm{max}}\right)$ was calculated using the Graph Pad program. Cell viability was shown to be unaltered when evaluated by means of the trypan blue exclusion test and Scepter device (Millipore, MA, USA).

\section{Membrane potential and mitochondrial mass}

Cells were incubated for $30 \mathrm{~min}$ in a fluorometer with the fluorescent probe Tetra-methyl-rhodamine methyl esther, $1 \times 10^{-7} \mathrm{~mol} / 1$ (TMRM) and mitochondrial membrane potential $\left(\Delta \Psi_{\mathrm{m}}\right)$ was evaluated using a Synergy Mx plate reader (BioTek Instruments, Winooski, VT, USA). Mitochondrial mass was measured in cells treated with the fluorescent dye 10-N-nonyl acridine orange (NAO, $5 \times$ $\left.10^{-6} \mathrm{~mol} / \mathrm{l}\right)$, which specifically binds to cardiolipin independently of $\Delta \Psi_{\mathrm{m}}(27)$.

\section{Measurement of ROS production}

Total ROS production was also assessed by fluorometry using a Synergy Mx plate reader (BioTek Instruments) following incubation (30 $\mathrm{min}$ ) with the fluorescent probe $\left(5 \times 10^{-6} \mathrm{~mol} / \mathrm{l}\right) 2^{\prime}, 7^{\prime}$-dichlorodihydrofluorescein diacetate (DCFH-DA), as described elsewhere (28). Hydrogen peroxide $\left(\mathrm{H}_{2} \mathrm{O}_{2}\right)$ was assessed with the Amplex $\operatorname{Red}^{\mathrm{R}}$ $\mathrm{H}_{2} \mathrm{O}_{2}$ /Peroxidase Assay kit.

\section{GSH content and proinflammatory cytokines}

Glutathione (GSH) content was evaluated following incubation (30 $\mathrm{min})$ with the fluorochrome 5-Chloromethylfluorescein Diacetate (CMFDA, $5 \times 10^{-6} \mathrm{~mol} / \mathrm{l}$ ) (19). In short, cells were seeded on 96-well plates, washed with PBS and then incubated with CMFDA diluted in PBS. After $15 \mathrm{~min}$ at $37^{\circ} \mathrm{C}$, fluorescence intensities were measured using excitation (492 nm) and emission (517 nm) wavelengths. The levels of intracellular GSH appeared as arbitrary units of fluorescence units. A Luminex 200 flow analyser system was employed to analyse IL6 and TNF $\alpha$ in serum from patients (Austin, TX, USA).

\section{Drugs and solutions}

Sodium cyanide, glutathione reductase, $N$-ethylmaleimide, bathophenanthroline disulfonic acid, nicotinamide adenine dinucleotide hydroxide $\mathrm{NADH}$, trypan blue, glucose, arginine and HBSS were supplied by Cambrex (Verviers, Belgium). $\mathrm{H}_{2} \mathrm{O}_{2}$, haemoglobin and RPMI 1640 medium supplemented with $20 \mathrm{mM}$ 4-(2-hydroxyethyl)-1piperazineethanesulfonic acid (HEPES) were obtained from Sigma-Aldrich. Dextran was acquired from Fluka (St Louis, MO, USA). DCFH diacetate was provided by Calbiochem (San Diego, CA). TMRM and CMFDA were supplied by Molecular Probes (Eugene, OR, USA). FicollPaque Plus was purchased from GE Healthcare (Little Chalfont, Buckinghamshire, UK). 


\section{Data analysis}

Statistical analysis was performed with SPSS 17.0 Software (SPSS Statistics, Inc.). Continuous variables were expressed as mean and S.D. or as median and 25th and 75th percentiles for parametric and non-parametric data respectively. The 12-week intervention period following completion of the metformin regimen in PCOS was evaluated using a paired Student's $t$-test and control vs PCOS data were evaluated using an unpaired Student's $t$-test. All the tests used a CI of $95 \%$ and differences were considered significant when $P<0.05$. Correlations were calculated using Pearson's correlation coefficient. Significant differences were considered when $P<0.05$.

\section{Results}

\section{Clinical and metabolic characteristics}

The clinical and metabolic characteristics of the subjects are presented in Table 1. No differences were obtained between values among controls at baseline and after 3 months (data not shown). In PCOS subjects at baseline there was a significant increase $(P<0.05)$ with respect to controls in waist circumference, systolic blood pressure,
hsCRP, insulin, HOMA-IR, testosterone, DHEA-S and androstendione and a significant decrease in HDLc and SHBG levels. Moreover, after treatment with metformin there was a decrease in glucose and FSH levels $(P<0.05)$ and an increase in DHEA-S $(P<0.05)$ when compared to baseline values.

\section{Mitochondrial $\mathrm{O}_{2}$ consumption}

First we monitored the rate of $\mathrm{O}_{2}$ consumption in PMNs from blood samples in an $\mathrm{O}_{2}$-tight chamber, as previously described $(19,29)$. The $\mathrm{O}_{2}$ requirement of the tissue was found to be mainly mitochondrial, since addition of sodium cyanide resulted in an almost complete (95-99\%) inhibition of $\mathrm{O}_{2}$ consumption (not shown). Figure $1 \mathrm{~A}$ shows how $\mathrm{O}_{2}$ consumption $(P<0.05)$ was lower among PCOS than controls at basal conditions, and illustrates the increase in $\mathrm{O}_{2}$ consumption after metformin treatment $(P<0.05)$, expressed as $\mathrm{nmol} \mathrm{O}_{2} /$ min per million cells.

\section{Membrane potential $\left(\Delta \psi_{m}\right)$ and mitochondrial mass}

The Fluoroskan plate reader revealed a significantly lower $(P<0.001)$ TMRM fluorescence in PCOS subjects in basal

Table 1 Anthropometric parameters, lipoprotein profile, hydrocarbonated metabolism parameters and circulating androgens of 41 control and 35 PCOS women treated with metformin during a 12-week period.

\begin{tabular}{l}
\hline \\
\hline Age (years) \\
Body weight (kg) \\
BMI (Kg/m²) \\
Waist circumference (cm) \\
Systolic BP (mmHg) \\
Diastolic BP (mmHg) \\
Total cholesterol (mg/dl) \\
LDLc (mg/dl) \\
HDLc (mg/dl) \\
Triglycerides (mg/dl) \\
Apolipoprotein A (mg/dl) \\
Apolipoprotein B (mg/dl) \\
hsCRP (mg/l) \\
Glucose (mg/dl) \\
Insulin $(\mu l U / m l)$ \\
HOMA-IR \\
FSH (mlU/ml) \\
LH (mlU/ml) \\
Testosterone (ng/ml) \\
DHEA-S ( $\mu \mathrm{g} / \mathrm{dl})$ \\
Androstenedione (ng/ml) \\
SHBG (nmol/l)
\end{tabular}

\begin{tabular}{c}
\hline Controls $(n=41)$ \\
\hline $26.2 \pm 4.4$ \\
$78.5 \pm 17.1$ \\
$29.6 \pm 6.5$ \\
$87.0 \pm 16.6^{*}$ \\
$110 \pm 11^{*}$ \\
$69 \pm 10$ \\
$175.0 \pm 32.6$ \\
$107.0 \pm 27.6$ \\
$51.3 \pm 13.4^{*}$ \\
$68.5(52.0 ; 97.3)$ \\
$142.8 \pm 30.6$ \\
$80.7 \pm 22.1$ \\
$2.76 \pm 2.20^{*}$ \\
$86.3 \pm 10.2$ \\
$9.8 \pm 5.6^{*}$ \\
$2.13 \pm 1.28^{*}$ \\
$4.38 \pm 2.44$ \\
$5.55 \pm 5.27$ \\
$0.44 \pm 0.21^{*}$ \\
$220.5 \pm 99.3^{*}$ \\
$2.42 \pm 0.97^{*}$ \\
$102.2 \pm 70.4^{*}$
\end{tabular}

\begin{tabular}{c}
\hline PCOS baseline $(n=35)$ \\
\hline $25.2 \pm 7.3$ \\
$83.7 \pm 23.6$ \\
$30.9 \pm 8.9$ \\
$95.2 \pm 17.1$ \\
$120 \pm 16$ \\
$73 \pm 13$ \\
$177.9 \pm 33.4$ \\
$114.1 \pm 27.5$ \\
$45.3 \pm 10.4$ \\
$82.5(49.8 ; 133.3)$ \\
$132.6 \pm 19.4$ \\
$85.3 \pm 24.2$ \\
$4.15 \pm 3.07$ \\
$85.5 \pm 10.5$ \\
$14.7 \pm 8.9$ \\
$3.16 \pm 2.12$ \\
$4.94 \pm 1.50$ \\
$6.40 \pm 5.04$ \\
$0.77 \pm 0.38$ \\
$353.2 \pm 175.7$ \\
$4.09 \pm 1.72$ \\
$53.7 \pm 37.9$
\end{tabular}

\begin{tabular}{c}
\hline PCOS metformin $(n=35)$ \\
\hline- \\
$83.4 \pm 23.7$ \\
$30.8 \pm 8.8$ \\
$95.4 \pm 15.8$ \\
$116 \pm 16$ \\
$72 \pm 11$ \\
$173.7 \pm 33.2$ \\
$109.4 \pm 27.8$ \\
$44.6 \pm 7.7$ \\
$75.0(58.8 ; 143.0)$ \\
$131.2 \pm 14.5$ \\
$84.9 \pm 27.0$ \\
$3.69 \pm 3.02$ \\
$81.5 \pm 10.5^{\dagger}$ \\
$13.4 \pm 8.6$ \\
$2.78 \pm 2.19$ \\
$4.25 \pm 1.74^{\dagger}$ \\
$5.53 \pm 3.38$ \\
$0.70 \pm 0.36$ \\
$398.9 \pm 206.8^{\dagger}$ \\
$3.56 \pm 1.63$ \\
$43.4 \pm 22.3$
\end{tabular}

Data are expressed as mean \pm s.D. for parametric data or as median (25th and 75 th percentiles) for non-parametric data. *Statistical significance $(P<0.05)$ was determined by an unpaired Student's $t$-test (control vs PCOS). ${ }^{\dagger}$ Statistical significance $(P<0.05)$ was determined by a paired Student's $t$-test (baseline vs 12 weeks). 

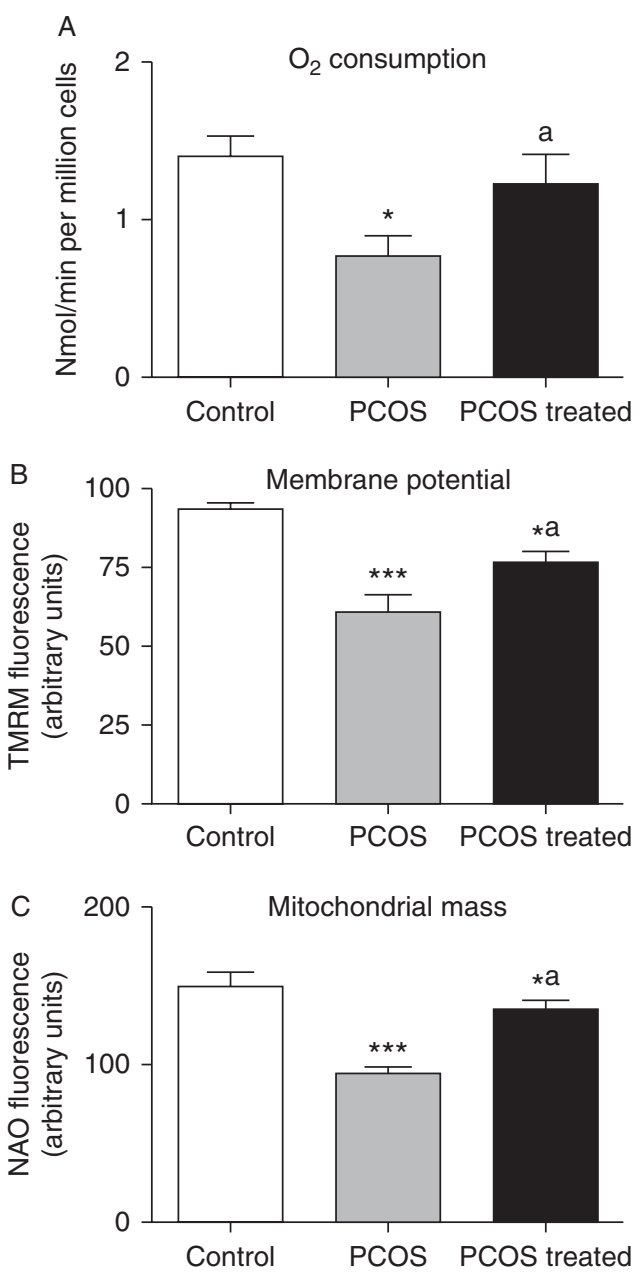

Figure 1

Effect of metformin treatment on mitochondrial function. (A) $\mathrm{O}_{2}$ consumption rate (measured as nanomol $\mathrm{O}_{2} / \mathrm{min} / \mathrm{million}$ cells), (B) membrane potential (measured by TMRM fluorescence), and (C) mitochondrial mass (measured by NAO fluorescence). ${ }^{*} P<005, * * * P<0.001$ vs control group. ${ }^{a} P<0.05$ vs $P C O S$ in basal conditions.

conditions $(P<0.05)$ and after treatment when compared to controls, and an increase in metformin-treated PCOS patients $(P<0.05)$ with respect to PCOS subjects in basal conditions that was indicative of a rise in $\Delta \psi_{m}$ (Fig. 1B). NAO fluorescence was also lower in the PCOS group in basal conditions $(P<0.001)$ and after treatment $(P<0.05)$ with respect to controls, and was higher in PCOS patients $(P<0.05)$ after metformin treatment than in basal conditions, thus indicating an increase in mitochondrial mass (Fig. 1C).

\section{ROS production}

ROS production was enhanced in PCOS in basal conditions $(P<0.01)$ and $(P<0.05)$ in the PCOS group with respect to controls. Metformin treatment induced a decrease in DCFH-DA fluorescence, demonstrating a decline in ROS production (Fig. $2 \mathrm{~A}, P<0.05$ ) in relation to baseline. $\mathrm{H}_{2} \mathrm{O}_{2}$ production was higher in PCOS subjects $(P<0.05)$ than in controls under basal conditions, and was lower among the former patients in treatment $(P<0.05)$ than at baseline (Fig. 2B). In contrast, CMFDA fluorescence was lower $(P<0.01)$ in the PCOS group than in controls
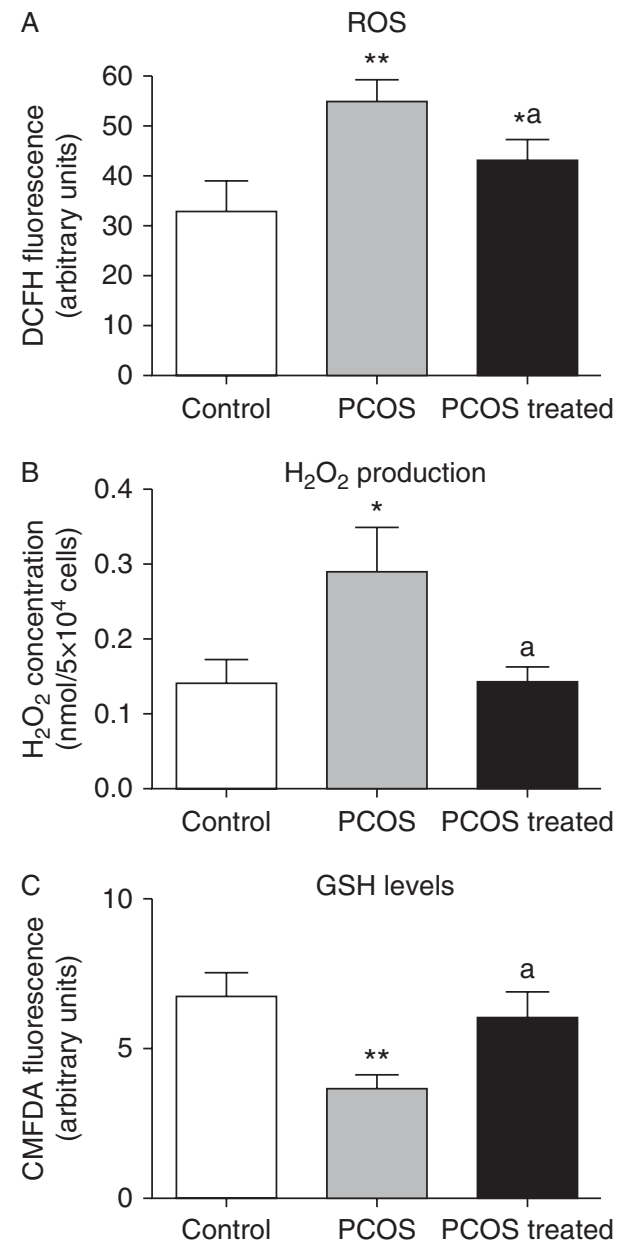

\section{Figure 2}

Effect of metformin treatment on oxidative stress. (A) ROS production (measured by DCFH fluorescence), (B) $\mathrm{H}_{2} \mathrm{O}_{2}$ (measured by the Amplex $\operatorname{Red}^{\mathrm{R}} \mathrm{H}_{2} \mathrm{O}_{2}$ /Peroxidase Assay kit), and (C) GSH content (measured by CMFDA fluorescence). ${ }^{*} P<005,{ }^{*} P<0.01$ vs control group. ${ }^{\mathrm{a}} P<0.05$ vs $\mathrm{PCOS}$ in basal conditions. 
under basal conditions, indicating a decrease in GSH levels (Fig. 2C), and was higher after treatment with PCOS $(P<0.05)$ than before treatment.

\section{IL6 and TNF $\alpha$ levels}

IL6 and TNF $\alpha$ levels $(\mathrm{pg} / \mathrm{ml})(P<0.01)$ were higher in the PCOS group in basal conditions, and $(P<0.05)$ in the PCOS-treated group with respect to the control group. After metformin treatment there was a decrease in IL6 and TNF $\alpha$ levels $(P<0.05)$ in comparison to basal conditions (Fig. 3A and $\mathrm{B}$ respectively).

\section{Correlation studies}

When we explored potential correlations among HOMAIR and mitochondrial function biomarkers, we found that the former was positively correlated with ROS production ( $r=0.486, P=0.025)$, GSH content $(r=0.710, P=0.049)$ and $\mathrm{H}_{2} \mathrm{O}_{2}(r=0.837, P=0.010)$, and negatively correlated with membrane potential $(r=-0.829, P=0.011)$ at baseline. These differences were not present after metformin treatment (Table 2).

\section{Discussion}

The aim of the present study was to throw light on the effects of treatment with metformin on mitochondrial function in PCOS patients. Specifically, we set out to demonstrate how this drug exerts beneficial effects on the leukocytes of these patients by evaluating endocrine and anthropometrical parameters and mitochondrial function. In comparison to controls, PCOS subjects exhibited higher values in waist circumference, systolic blood pressure, hsCRP, testosterone, DHEA-S and
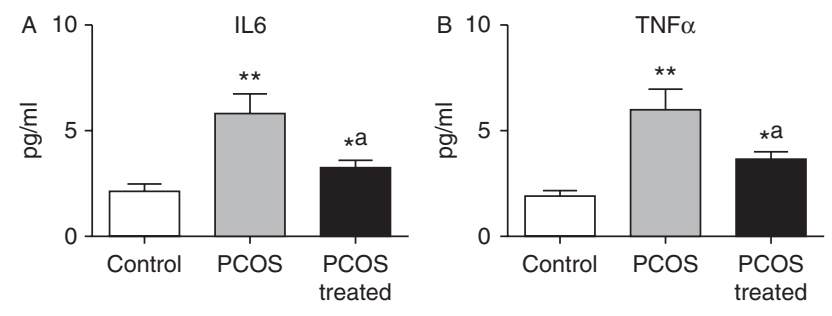

\section{Figure 3}

Effect of metformin treatment on levels of IL6 (A) and TNF $\alpha$ (B); both were evaluated with the Luminex 200 flow analyser system. ${ }^{*} P<005, * * P<0.01$ vs control group. ${ }^{a} P<0.05$ vs $P C O S$ in basal conditions.
Table 2 Correlation coefficients between HOMA-IR and mitochondrial function biomarkers in PCOS women.

\begin{tabular}{|c|c|c|c|c|}
\hline & \multicolumn{4}{|c|}{ HOMA-IR } \\
\hline & \multicolumn{2}{|c|}{ PCOS baseline } & \multicolumn{2}{|c|}{ PCOS metformin } \\
\hline & $r$ & $P$ & $r$ & $P$ \\
\hline $\begin{array}{l}\text { Mitochondrial } \mathrm{O}_{2} \\
\text { consumption }\end{array}$ & -0.545 & 0.163 & -0.510 & 0.197 \\
\hline $\begin{array}{l}\text { Membrane } \\
\text { potential }\end{array}$ & -0.829 & 0.011 & -0.406 & 0.318 \\
\hline $\begin{array}{l}\text { Mitochondrial } \\
\text { mass }\end{array}$ & 0.108 & 0.799 & 0.262 & 0.531 \\
\hline $\begin{array}{l}\text { Reactive oxygen } \\
\text { species }\end{array}$ & 0.486 & 0.025 & 0.212 & 0.614 \\
\hline GSH content & 0.710 & 0.049 & 0.409 & 0.314 \\
\hline $\mathrm{H}_{2} \mathrm{O}_{2}$ & 0.837 & 0.010 & 0.094 & 0.825 \\
\hline
\end{tabular}

Correlation coefficients were estimated by Pearson's correlation for all parameters. Data are shown as $r$ correlation coefficient and $P$ value.

androstendione, and lower levels of HDLc, SHBG, insulin and HOMA-IR, thereby suggesting the appearance of insulin resistance. We have observed how metformin reduces glucose and FSH in PCOS subjects, increases mitochondrial $\mathrm{O}_{2}$ consumption, membrane potential, mitochondrial mass and GSH levels, and decreases production of ROS and $\mathrm{H}_{2} \mathrm{O}_{2}$. Moreover, we have also seen how metformin induces a decrease in IL6 and TNF $\alpha$ levels in leukocytes from these patients. In addition, we demonstrate that HOMA-IR and mitochondrial function biomarkers are positively correlated with ROS production, GSH content and $\mathrm{H}_{2} \mathrm{O}_{2}$, and negatively correlated with membrane potential at baseline. These differences are not present after metformin treatment.

It has been shown that the leukocytes of women with PCOS are in a proinflammatory state due in part to high levels of glucose and plasma hsCRP (30). We have previously reported an inhibition of mitochondrial $\mathrm{O}_{2}$, membrane potential and antioxidant levels, an impairment of complex I activity and an increase in ROS production in leukocytes from these women (19). In the present work, we demonstrate that metformin exerts beneficial effects by increasing mitochondrial $\mathrm{O}_{2}$ consumption, membrane potential and mitochondrial mass in PCOS patients. In line with the present results, it has recently been shown that 2 -week treatment with metformin enhances mitochondrial respiration in mitochondrial-deficient AMP kinase mice (31).

We have previously demonstrated that insulin resistance in women with PCOS can stimulate ROS production by leukocytes (19). Our present results show that ROS production decreases after a 12-week treatment with 
metformin. In this sense, there are several studies that show that the beneficial effect of metformin on mitochondrial oxidative stress occurs through a decline in ROS production $(16,32)$, or, according to a previous in vitro study, through activation of AMP kinase and mitochondrial biogenesis (33). This evidence may explain, in part, the beneficial effects on mitochondrial membrane potential and mitochondrial mass that we have observed after treatment with metformin. In line with this idea, other studies have demonstrated that metformin enhances the expression of PGC1 $\alpha$, a key regulator of mitochondrial biogenesis (34), and the activity of mitochondrial enzymes (35). Furthermore, it has recently been described that metformin suppresses glunoneogenesis by inhibiting mitochondrial glycerophosphate dehydrogenase, which identifies it as a novel therapeutic target for insulin resistance treatment (36).

Leukocytes are highly sensitive to the oxidative damage mediated by ROS released from different types of cells and sources (37). Paradoxically, they are implicated in the generation of ROS, high levels of which are harmful to cells, as they trigger lipid peroxidation and cell death (38). These deleterious effects of ROS are counteracted by the organism's complex antioxidant system, including GSH, which plays a key role in protecting cells from oxidative stress. Therefore, impaired ROS production, GSH levels and mitochondrial membrane potential - all characteristic of PCOS - represent dysfunction within the respiratory chain, which compromises the function of the mitochondria as a source of energy. This aspect of PCOS has been the focus of little attention until now, but our findings provide evidence that the beneficial effects of metformin are a result of an improvement in the different mitochondrial parameters that we have evaluated. In this context, other studies have demonstrated a protective effect of metformin against oxidative stress - for example, that induced by doxorubicin in cardiomyocytes - through its modulation of the ferritin heavy chain (FHC), the main iron-storage protein (39). In addition, metformin has demonstrated beneficial effects on mitochondrial function in both the heart and the brain $(40,41)$.

Finally, we explored potential correlations between HOMA-IR and mitochondrial function biomarkers in PCOS and found that HOMA-IR was positively correlated with ROS production, GSH content and $\mathrm{H}_{2} \mathrm{O}_{2}$, and was negatively correlated with membrane potential at baseline. These results suggest that HOMA-IR is related to mitochondrial function in PCOS and these differences disappeared after metformin treatment.
Leukocytes can also release proinflammatory cytokines such as TNF $\alpha$ and IL6, which play an important role in the development of IR in PCOS patients (19). The present study demonstrates that metformin decreases TNF $\alpha$ and IL6 levels, which would represent a beneficial effect, as an augmented release of proinflammatory cytokines following activation by ROS-induced oxidative stress is thought to inhibit insulin signalling and impair glucose uptake (18). It has been suggested that metformin inhibits the activation of NF- $\mathrm{KB}$ in HUVEC exposed to inflammatory cytokines, thereby reducing the expression of genes that encode adhesion and proinflammatory molecules (42). In line with this idea, Huang et al. (43) have demonstrated that metformin inhibits IL6 production and activation of the TNF $\alpha$-induced NF- $\kappa \mathrm{B}$ pathway.

The present study has some limitations, including the size of the study population. In addition, our results do not throw light on the mechanism by which metformin can suppress mitochondrial glycerol-3-phosphate dehydrogenase in leukocytes from PCOS patients, which needs to be explored in future research.

In conclusion, the present study demonstrates that metformin can exert beneficial effects on mitochondrial function in PCOS patients and helps to control levels of proinflammatory cytokines. Future studies of mitochondria in these circumstances may uncover the molecular mechanism underlying metformin's efficacy in ameliorating IR in PCOS patients, thereby helping to develop strategies to reduce the risk of the onset of type 2 diabetes in women with this syndrome.

\section{Declaration of interest}

The authors declare that there is no conflict of interest that could be perceived as prejudicing the impartiality of the research reported.

\section{Funding}

This study was financed by grant numbers PI13/1025, PI12/1984, PI13/0073, CIBERehd CB06/04/0071, PROMETEO 2010/060, UGP-14-93, UGP-14-95 and by the European Regional Development Fund (ERDF 'A way to build Europe'). V M Víctor and $M$ Rocha are recipients of contracts from the Ministry of Health of the Valencian Regional Government and Carlos III Health Institute (CES10/030 and CP10/0360 respectively). S Rovira-Llopis and $\mathrm{N}$ Diaz-Morales is the recipient of a postgraduate fellowship from Carlos III Health Institute (FI11/00637 and FI14/00125, respectively). C Bañuls is the recipient of a Sara Borrell contract from Carlos III Health Institute (CD14/00043).

\section{Author contribution statement}

The authors' responsibilities were as follows: A Hernández-Mijares conducted the study. M Gómez provided overall supervision and patient 
follow-up. S Rovira-Llopis, C Bañuls, N Diaz-Morales, R Castelló and $\mathrm{R}$ Falcón performed the laboratory analyses and collected data. M Rocha assisted in the design of the experiment and provided support throughout the course of the study and analysis. V M Víctor, A Hernández-Mijares, $S$ Rovira-Llopis, C Bañuls and M Rocha performed statistical analyses, interpreted the data and prepared the manuscript. A Hernández-Mijares and $\mathrm{V} \mathrm{M}$ Víctor were responsible for its final content.

\section{Acknowledgements}

We thank Isabel Soria for her work in the extraction of biological samples (University Hospital Doctor Peset) and Brian Normanly for his editorial assistance (University of Valencia).

\section{References}

1 Diamanti-Kandarakis E, Kouli CR, Bergiele AT, Filandra FA, Tsianateli TC, Spina GG, Zapanti ED \& Bartzis M. A survey of the polycystic ovary syndrome in the Greek island of Lesbos: hormonal and metabolic profile. Journal of Clinical Endocrinology and Metabolism 1999 84 4006-4011. (doi:10.1210/jcem.84.11.6148)

2 Asunción M, Calvo RM, San Millán JL, Sancho J, Avila S \& EscobarMorreale HF. A prospective study of the prevalence of the polycystic ovary syndrome in unselected Caucasian women from Spain. Journal of Clinical Endocrinology and Metabolism 200085 2434-2438. (doi:10.1210/ jcem.85.7.6682)

3 Dunaif A, Segal KR, Futterweit W \& Dobrjansky A. Profound peripheral insulin resistance, independent of obesity, in polycystic ovary syndrome. Diabetes 198938 1165-1174. (doi:10.2337/diab.38.9.1165)

4 Legro RS, Kunselman AR, Dodson WC \& Dunaif A. Prevalence and predictors of risk for type 2 diabetes mellitus and impaired glucose tolerance in polycystic ovary syndrome: a prospective, controlled study in 254 affected women. Journal of Clinical Endocrinology and Metabolism 199984 165-169. (doi:10.1210/jcem.84.1.5393)

5 Ehrmann DA, Liljenquist DR, Kasza K, Azziz R, Legro RS, Ghazzi MN \& PCOS/Troglitazone Study Group . Prevalence and predictors of the metabolic syndrome in women with polycystic ovary syndrome. Journal of Clinical Endocrinology and Metabolism 200691 48-53. (doi:10.1210/jc.2005-1329)

6 Diamanti-Kandarakis E, Christakou CD, Kandaraki E \& Economou FN. Metformin: an old medication of new fashion: evolving new molecular mechanisms and clinical implications in polycystic ovary syndrome. European Journal of Endocrinology 2010162 193-212. (doi:10.1530/ EJE-09-0733)

7 Palomba S, Falbo A, Zullo F \& Orio F Jr. Evidence-based and potential benefits of metformin in the polycystic ovary syndrome: a comprehensive review. Endocrine Reviews 200930 1-50. (doi:10.1210/er. 2008-0030)

8 Johnson NP. Metformin use in women with polycystic ovary syndrome. Annals of Transplantation 20142 56. (doi:10.3978/j.issn.2305-5839. 2014.04.15)

9 Shulman GI. Cellular mechanisms of insulin resistance. Journal of Clinical Investigation 2000106 171-176. (doi:10.1172/JCI10583)

10 Lowell BB \& Shulman GI. Mitochondrial dysfunction and type 2 diabetes. Science 2005307 384-387. (doi:10.1126/science.1104343)

11 Larsen S, Stride N, Hey-Mogensen M, Hansen CN, Andersen JL, Madsbad S, Worm D, Helge JW \& Dela F. Increased mitochondrial substrate sensitivity in skeletal muscle of patients with type 2 diabetes. Diabetologia 201154 1427-1436. (doi:10.1007/s00125-011-2098-4)

12 Irving BA, Short KR, Nair KS \& Stump CS. Nine days of intensive exercise training improves mitochondrial function but not insulin action in adult offspring of mothers with type 2 diabetes. Journal of
Clinical Endocrinology and Metabolism 201196 E1137-E1141. (doi:10.1210/jc.2010-2863)

13 Owen MR, Doran E \& Halestrap AP. Evidence that metformin exerts its anti-diabetic effects through inhibition of complex 1 of the mitochondrial respiratory chain. Biochemical Journal 2000348 607-614. (doi:10.1042/bj3480607)

14 Brunmair B, Staniek K, Gras F, Scharf N, Althaym A, Clara R, Roden M, Gnaiger E, Nohl H, Waldhäusl W et al. Thiazolidinediones like metformin inhibit respiratory complex I: a common mechanism contributing to their antidiabetic actions? Diabetes 200453 1052-1059. (doi:10.2337/diabetes.53.4.1052)

15 Carvalho C, Correia S, Santos MS, Seiça R, Oliveira CR \& Moreira PI. Metformin promotes isolated rat liver mitochondria impairment. Molecular and Cellular Biochemistry 2008308 75-83. (doi:10.1007/ s11010-007-9614-3)

16 Kane DA, Anderson EJ III, Price JW, Woodlief TL, Lin CT, Bikman BT, Cortright RN \& Neufer PD. Metformin selectively attenuates mitochondrial $\mathrm{H}_{2} \mathrm{O}_{2}$ emission without affecting respiratory capacity in skeletal muscle of obese rats. Free Radical Biology \& Medicine 201049 1082-1087. (doi:10.1016/j.freeradbiomed.2010.06.022)

17 Hawley SA, Gadalla AE, Olsen GS \& Hardie DG. The antidiabetic drug metformin activates the AMP-activated protein kinase cascade via an adenine nucleotide-independent mechanism. Diabetes 200251 2420-2425. (doi:10.2337/diabetes.51.8.2420)

18 Gonzalez F, Rote NS, Minium J \& Kirwan JP. Reactive oxygen speciesinduced oxidative stress in the development of insulin resistance and hyperandrogenism in polycystic ovary syndrome. Journal of Clinical Endocrinology and Metabolism 200691 336-340. (doi:10.1210/jc.2005-1696)

19 Victor VM, Rocha M, Bañuls C, Sanchez-Serrano M, Sola E, Gomez M \& Hernandez-Mijares A. Mitochondrial complex I impairment in leukocytes from polycystic ovary syndrome patients with insulin resistance. Journal of Clinical Endocrinology and Metabolism 200994 3505-3512. (doi:10.1210/jc.2009-0466)

20 Victor VM, Rocha M, Bañuls C, Alvarez A, de Pablo C, SanchezSerrano M, Gomez M \& Hernández-Mijares A. Induction of oxidative stress and human leukocyte/endothelial cell interactions in polycystic ovary syndrome patients with insulin resistance. Journal of Clinical Endocrinology and Metabolism 201196 3115-3122. (doi:10.1210/jc. 2011-0651)

21 Gonzalez F, Rote NS, Minium J \& Kirwan JP. Increased activation of nuclear factor $\mathrm{\kappa B}$ triggers inflammation and insulin resistance in polycystic ovary syndrome. Journal of Clinical Endocrinology and Metabolism 200691 1508-1512. (doi:10.1210/jc.2005-2327)

22 Gonzalez F, Rote NS, Minium J \& Kirwan JP. In vitro evidence that hyperglycemia stimulates tumor necrosis factor $\alpha$ release in obese women with polycystic ovary syndrome. Journal of Endocrinology 2006 188 521-529. (doi:10.1677/joe.1.06579)

23 Kocer D, Bayram F \& Diri H. The effects of metformin on endothelial dysfunction lipid metabolism and oxidative stress in women with polycystic ovary syndrome. Gynecological Endocrinology 201430 367-371. (doi:10.3109/09513590.2014.887063)

24 Pavlovic D, Kocic R, Kocic G, Jevtovic T, Radenkovic S, Mikic D, Stojanovic M \& Djordjevic PB. Effect of four-week metformin treatment on plasma and arythrocyte antioxidative defense enzymes in newly diagnosed obese patients with type 2 diabetes. Diabetes, Obesity \& Metabolism 20002 251-256. (doi:10.1046/j.1463-1326.2000.00089.x)

25 The Rotterdam ESHRE/ASRM-Sponsored PCOS Consensus Workshop Group. Revised 2003 consensus on diagnostic criteria and long-term health risks related to polycystic ovary syndrome. Fertility and Sterility 200481 19-25. (doi:10.1016/j.fertnstert.2003.10.004)

26 Azziz R, Sanchez LA, Knochenhauer ES, Moran C, Lazenby J, Stephens KC, Taylor K \& Boots LR. Androgen excess in women: experience with over 1000 consecutive patients. Journal of Clinical Endocrinology and Metabolism 200489 453-462. (doi:10.1210/jc. 2003-031122) 
27 Hernandez-Mijares A, Rocha M, Rovira-Llopis S, Bañuls C, Bellod L, de Pablo C, Alvarez A, Roldan-Torres I, Sola-Izquierdo E \& Victor VM. Human leukocyte/endothelial cell interactions and mitochondrial dysfunction in type 2 diabetic patients and their association with silent myocardial ischemia. Diabetes Care 201336 1695-1702. (doi:10.2337/ dc12-1224)

28 Rovira-Llopis S, Rocha M, Falcon R, de Pablo C, Alvarez A, Jover A, Hernandez-Mijares A \& Victor VM. Is myeloperoxidase a key component in the ROS-induced vascular damage related to nephropathy in type 2 diabetes? Antioxidants \& Redox Signaling 201319 1452-1458. (doi:10.1089/ars.2013.5307)

29 Hernandez-Mijares A, Rocha M, Apostolova N, Borras C, Jover A, Bañuls C, Sola E \& Victor VM. Mitochondrial complex I impairment in leukocytes from type 2 diabetic patients. Free Radical Biology \& Medicine 201150 1215-1221. (doi:10.1016/j.freeradbiomed.2011.01.019)

30 Kelly CC, Lyall H, Petrie JR, Gould GW, Connell JM \& Sattar N. Low grade chronic inflammation in women with polycystic ovarian syndrome. Journal of Clinical Endocrinology and Metabolism 200186 2453-2455. (doi:10.1210/jcem.86.6.7580)

31 Kristensen JM, Larsen S, Helge JW, Dela F \& Wojtaszewski JF. Two weeks of metformin treatment enhances mitochondrial respiration in skeletal muscle of AMPK kinase dead but not wild type mice. PLoS ONE 20138 e53533. (doi:10.1371/journal.pone.0053533)

32 Hou X, Song J, Li XN, Zhang L, Wang X, Chen L \& Shen YH. Metformin reduces intracellular reactive oxygen species levels by upregulating expression of the antioxidant thioredoxin via the AMPK-FOXO3 pathway. Biochemical and Biophysical Research Communications 2010 396 199-205. (doi:10.1016/j.bbrc.2010.04.017)

33 Kukidome D, Nishikawa T, Sonoda K, Imoto K, Fujisawa K, Yano M, Motoshima H, Taguchi T, Matsumura T \& Araki E. Activation of AMP-activated protein kinase reduces hyperglycemia-induced mitochondrial reactive oxygen species production and promotes mitochondrial biogenesis in human umbilical vein endothelial cells. Diabetes 200655 120-127. (doi:10.2337/diabetes.55.01.06.db05-0943)

34 Wu Z, Puigserver P, Andersson U, Zhang C, Adelmant G, Mootha V, Troy A, Cinti S, Lowell B, Scarpulla RC et al. Mechanisms controlling mitochondrial biogenesis and respiration through the thermogenic coactivator PGC-1. Cell 199998 115-124. (doi:10.1016/S00928674(00)80611-X)
35 Suwa M, Egashira T, Nakano H, Sasaki H \& Kumagai S. Metformin increases the PGC- $1 \alpha$ protein and oxidative enzyme activities possibly via AMPK phosphorylation in skeletal muscle in vivo. Journal of Applied Physiology 2006101 1685-1692. (doi:10.1152/japplphysiol. 00255.2006)

36 Madiraju A, Erion DM, Rahimi Y, Zhang XM, Braddock D, Albright RA, Prigaro BJ, Wood JL, Bhanot S, MacDonald MJ et al. Metformin suppresses glunoneogenesis by inhibiting mitochondrial glycerophosphate dehydrogenase. Nature 2014510 542-546. (doi:10.1038/nature13270)

37 De la Fuente M \& Victor VM. Antioxidants as modulators of immune function. Immunology and Cell Biology 200078 49-54. (doi:10.1046/j. 1440-1711.2000.00884.x)

38 Victor VM, Rocha M \& De la Fuente M. Immune cells: free radicals and antioxidants in sepsis. International Immunopharmacology 20044 327-347. (doi:10.1016/j.intimp.2004.01.020)

39 Asensio-Lopez MC, Sanchez-Mas J, Pascual-Figal DA, de Torre C, Valdes $\mathrm{M} \&$ Lax A. Ferrin heavy chain as main mediator of preventive effect of metformin against mitochondrial damage induced by doxorubicin in cardiomyocytes. Free Radical Biology \& Medicine 201467 19-29. (doi:10.1016/j.freeradbiomed.2013.11.003)

40 Pintana H, Apaijai N, Pratchayasakul W, Chattipakorn N \& Chattipakorn SC. Effects of metformin on learning and memory behaviors and brain mitochondrial functions in high fat diet induced insulin resistant rats. Life Sciences 201291 409-414. (doi:10.1016/j.lfs. 2012.08.017)

41 Apaijai N, Pintana H, Chattipakorn SC \& Chattipakorn N. Cardioprotective effects of metformin and vildagliptin in adult rats with insulin resistance induced by a high-fat diet. Endocrinology 2012153 3878-3885. (doi:10.1210/en.2012-1262)

42 Hattori Y, Suzuki K, Hattori S \& Kasai K. Metformin inhibits cytokineinduced nuclear factor $\kappa \mathrm{B}$ activation via AMP-activated protein kinase activation in vascular endothelial cells. Hypertension $2006 \mathbf{4 7}$ 1183-1188. (doi:10.1161/01.HYP.0000221429.94591.72)

43 Huang NL, Chiang SH, Hsueh CH, Liang YJ, Chen YJ \& Lai LP.

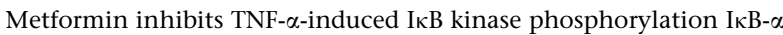
degradation and IL-6 production in endothelial cells through PI3Kdependent AMPK phosphorylation. International Journal of Cardiology 2009134 169-175. (doi:10.1016/j.ijcard.2008.04.010)
Received 8 June 2015

Revised 31 July 2015

Accepted 27 August 2015 